\title{
Correction to: Multilayered-quality education ecosystem (MQEE): an intelligent education modal for sustainable quality education
}

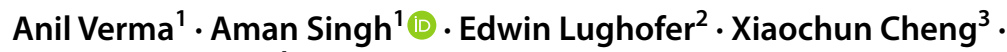 \\ Khalid Abualsaud ${ }^{4}$
}

Published online: 20 August 2021

(c) Springer Science+Business Media, LLC, part of Springer Nature 2021

\section{Correction to: Journal of Computing in Higher Education https://doi.org/10.1007/s12528-021-09291-1}

The original version of this article unfortunately contained an error and it has been corrected with this erratum.

The Acknowledgements section was missing from this article and should have read "The third author acknowledges the support by the Austrian Science Fund (FWF): Contract Number P32272-N38, acronym IL-EFS".

Publisher's Note Springer Nature remains neutral with regard to jurisdictional claims in published maps and institutional affiliations.

The original article can be found online at https://doi.org/10.1007/s12528-021-09291-1.

Aman Singh

amansingh.x@gmail.com

Anil Verma

anilverma.iiitb@gmail.com

Edwin Lughofer

Edwin.Lughofer@jku.at

Xiaochun Cheng

xiaochun.cheng@gmail.com

Khalid Abualsaud

k.abualsaud@qu.edu.qa

1 Department of Computer Science and Engineering, Lovely Professional University, Phagwara, Punjab 144411, India

2 Department of Knowledge-Based Mathematical Systems, Johannes Kepler University Linz, 69A-4040 Linz, Austria

3 Department of Computer Science, Middlesex University, London NW4 4BT, UK

4 Department of Computer Science, Qatar University, Doha, Qatar 